\title{
Synthesis and biological evaluation of mono- and tri-heterocyclic azole derivatives as anticancer agents
}

\author{
Agnieszka Zagórska a* , Anna Czopek ${ }^{a}$, Magdalena Mielczarek-Puta ${ }^{b}$, Marta Struga ${ }^{\text {}}$, Marek Bajda ${ }^{c}$ \\ aDepartment of Medicinal Chemistry Jagielonian University Medical College \\ ${ }^{b}$ Department of Biochemistry, Medical University of Warsaw \\ 'Department of Physicochemical Drug Analysis, Jagiellonian University Medical College
}

\section{- Background:}

Angiogenesis is well known as a vital step in the process of cancer growth. Thymidine phosphorylase (TP) is identical to the angiogenic factor platelet-derived endothelial-cell growth factor (PD-ECGF) and catalyzes the conversion of thymidine to thymine and 2-deoxy- $\alpha$-D-ribose-1phosphate (dRib-1-P). TP is overexpressed in several human cancers, has been shown to promote tumor angiogenesis, invasion, metastasis, evasion of the immune response and resistance to apoptosis. Given its various biological functions in cancer progression, TP is a promising target in cancer treatment. TP inhibitors (TPIs) suppress the formation of new blood vessels and stop tumor growth. The most potent TPIs known up to now is tipiracil (5-chloro-6-[1-(2-iminopyrrolidinyl)methyl] uracil hydrochloride), while 7-deazaxanthine (7DX) is the first purine analog classified as a TP inhibitor.

\section{- Objectives:}

The aim of the presented work was to biologically evaluate a series of synthesized mono- and tri-heterocyclic azole derivatives. Acetylphenylpiperazin-1-yl-alkyl derivatives of spiro[imidazolidine-naphthalene/indene]-2,5-dione, imidazo[2,1-f]purine-2,4-dione and pyrimido[2,1-f]purine1,3,9-trione were designed as structural counterparts of tipiracil and 7-deazaxanthine, potent thymidine phosphorylase inhibitors (TPIs) and evaluated for their cytotoxicity against a series of human cancer cell lines as well as normal human HaCaT and Chinese hamster fibroblast cell lines.

Imidazolidine and cyclic guanidine scaffold
- Pyrimidine ring from 7-deazaxanthine and
tipiracil

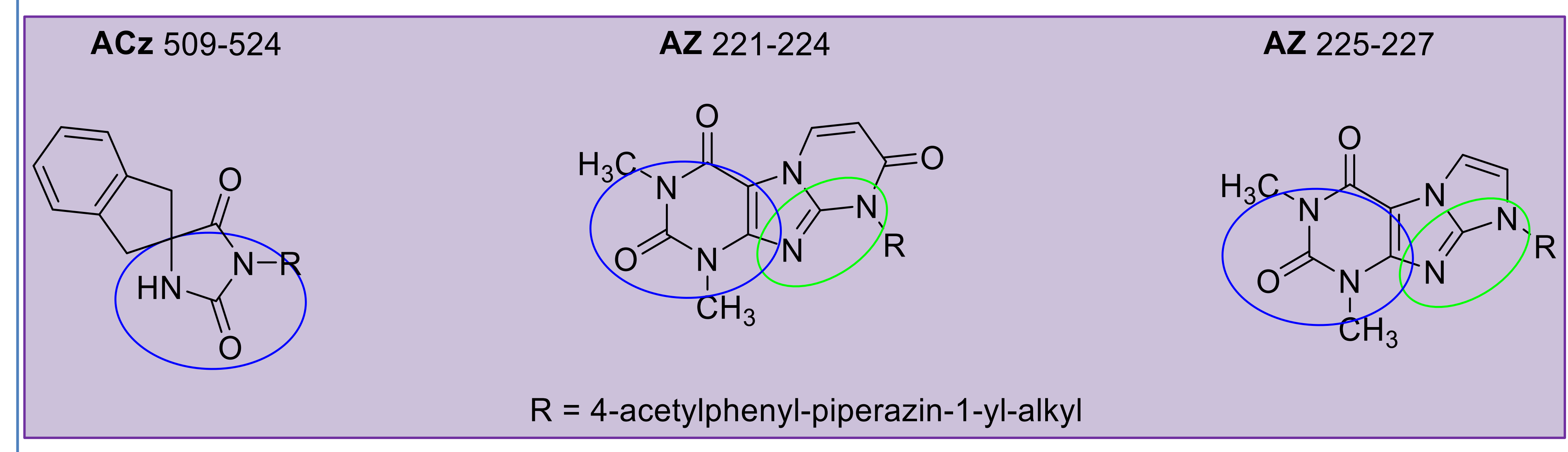

\section{- Methods:}

The library of compounds was obtained via a multistep synthesis according to the synthetic route published elsewhere [1,2]. TP inhibitory activity was determined spectrophotometrically according to a modified method of Krenitsky [3]. To establish their cytotoxic effects, the compounds were screened for their in vitro antiproliferative activity against a panel of different human cancer cell lines, namely prostate (PC3) and colon (SW480, SW620), in contrast to normal cell lines - human immortalized keratinocytes (HaCaT) and Chinese hamster fibroblast (V79), by the MTT method, using doxorubicin as a reference compound.

- Results:

The anticancer profile of the tested derivatives, expressed as $I C_{50}$ values, showed that the series of spiro[imidazolidine-naphthalene/indene]-2,5dione derivatives was incomparably more potent than the purine derivatives. The most active compound, ACz 512 (1-(5-(4-(4acetylphenyl)piperazin-1-yl)pentyl)-3',4'-dihydro-2'H-spiro[imidazolidine-4,1'-naphthalene]-2,5-dione), exhibited growth-inhibitory potency on SW480 $\left(\mathrm{IC}_{50}=16.8 \pm 2.37 \mu \mathrm{M}\right)$, SW620 $\left(\mathrm{IC}_{50}=12.9 \pm 3.16 \mu \mathrm{M}\right)$ and PC3 $\left(\mathrm{IC}_{50}=20.58 \pm 3.35 \mu \mathrm{M}\right)$. Furthermore, the highest selectivity index $(\mathrm{SI})$ was achieved for compound ACz 512. Evaluation of potential thymidine phosphorylase inhibitory activity from the selected compounds demonstrated that ACz 512 exhibited $21.21 \%$ inhibitory activity at $100 \mu \mathrm{M}$.

\begin{tabular}{|c|c|c|c|c|c|c|c|c|}
\hline \multirow{3}{*}{ Compound } & \multicolumn{7}{|c|}{ Cell lines } & \multirow{3}{*}{$\begin{array}{c}\text { V } 79 \\
I C_{50}(\mu M)\end{array}$} \\
\hline & \multicolumn{2}{|c|}{ SW480 } & \multicolumn{2}{|c|}{ SW 620} & \multicolumn{2}{|l|}{ PC3 } & \multirow{2}{*}{$\begin{array}{c}\text { НаСаT } \\
I C_{50}(\mu \mathrm{M})\end{array}$} & \\
\hline & $I_{50}(\mu \mathrm{M})$ & SI & $I_{50}(\mu \mathrm{M})$ & SI & $I_{50}(\mu \mathrm{M})$ & SI & & \\
\hline ACz 509 & $54.18 \pm 3.89$ & 0.77 & $67.05 \pm 5.24$ & 0.62 & $57.62 \pm 5.86$ & 0.72 & $41.75 \pm 7.62$ & $106.57 \pm 10.96$ \\
\hline $\mathrm{ACz} 510$ & $40.68 \pm 3.54$ & 0.55 & $43.12 \pm 4.41$ & 0.52 & $36.23 \pm 4.98$ & 0.62 & $22.55 \pm 2.69$ & $59.39 \pm 6.72$ \\
\hline $\mathrm{ACz} 511$ & $129.35 \pm 10.85$ & 0.88 & $104.15 \pm 7.36$ & 1.09 & $64.45 \pm 6.76$ & 1.76 & $113.53 \pm 8.93$ & $63.6 \pm 6.73$ \\
\hline $\mathrm{ACz} 512$ & $16.8 \pm 2.37$ & 2.19 & $12.9 \pm 3.16$ & 2.85 & $20.58 \pm 3.35$ & 1.79 & $36.78 \pm 5.32$ & $32.98 \pm 4.13$ \\
\hline$A C z 521$ & $102.09 \pm 2.37$ & - & $>200$ & - & $>200$ & - & $>200$ & $171.64 \pm 18.21$ \\
\hline$A C z 522$ & $53.67 \pm 7.78$ & 2.95 & $78.34 \pm 4.76$ & 2.02 & $95.12 \pm 8.4$ & 1.67 & $158.51 \pm 14.12$ & $103.87 \pm 15.43$ \\
\hline $\mathrm{ACz} 523$ & $103.33 \pm 9.36$ & 1.61 & $79.92 \pm 5.12$ & 2.08 & $88.9 \pm 3.64$ & 1.87 & $166.61 \pm 1.58$ & $38.76 \pm 1,01$ \\
\hline$A C z 524$ & $62.17 \pm 7.48$ & 1.42 & $84.3 \pm 4.87$ & 1.05 & $42.8 \pm 3.64$ & 2.06 & $88.32 \pm 3.27$ & $61.28 \pm 5.34$ \\
\hline Az 221 & $>200$ & - & $169.28 \pm 22.91$ & 0.49 & $71.85 \pm 9.85$ & 1.17 & $83.91 \pm 4.2$ & $46.87 \pm 9.53$ \\
\hline Az 222 & $>200$ & - & $>200$ & - & $94.2 \pm 8.36$ & 1.33 & $125.84 \pm 14.35$ & $38.1 \pm 5.76$ \\
\hline Az 223 & $>200$ & - & $>200$ & - & $90.97 \pm 5.86$ & 1.06 & $96.45 \pm 4.93$ & $40.92 \pm 5.39$ \\
\hline AZ 225 & $153.34 \pm 11.29$ & 0.47 & $144.64 \pm 3.76$ & 0.5 & $101.26 \pm 15.37$ & 0.72 & $72.78 \pm 6.08$ & $54.46 \pm 8.0$ \\
\hline Az 226 & $>200$ & - & $97.69 \pm 10.74$ & 0.6 & $60.28 \pm 0.4$ & 0.97 & $58.65 \pm 4.91$ & $44.59 \pm 0.43$ \\
\hline Az 227 & $>200$ & - & $130.67 \pm 12.64$ & 0.73 & $86.12 \pm 9.85$ & 1.11 & $96.06 \pm 5.32$ & $68.85 \pm 4.86$ \\
\hline Doxorubicin* & $0.29 \pm 0.08$ & 0.81 & $0.31 \pm 0.07$ & 0.76 & $0.59 \pm 0.02$ & 0.4 & $0.235 \pm 0.03$ & $2.01 \pm 0.03$ \\
\hline
\end{tabular}

\section{- Conclusions:}

The data allowed the identification of a lead-like structure for anticancer activity based on TP inhibition.

1. Zagórska A., Kołaczkowski M., Bucki A. et al. Eur J Med Chem. 97 (2015) 142-154, 2.Czopek A., Kołaczkowski M., Bucki A. et al. Bioorg Med Chem. 23:13 (2015) 3436-3447, 3. Shahzad S.A., Yar M., Bajda M. et al. Bioorg Med Chem. 22 (2014) 1008-1015.

The project was financially supported by Jagiellonian University Medical College funding grant no N42/DBS/000178 